\title{
Investigation into the Thermal Behavior and Loadability Characteristic of a YASA-AFPM Generator via an Improved 3-D Coupled Electromagnetic-Thermal Approach
}

\author{
Saadat Jamali Arand ${ }^{1, *}$, Amir Akbari ${ }^{2}$, Mohammad Ardebili ${ }^{2}$ \\ ${ }^{1}$ Electrical Engineering Department, Faculty of Engineering, Yasouj University, Yasouj, Iran \\ ${ }^{2}$ Department of Electrical and Computer Engineering, K. N. Toosi University of Technology, Tehran, Iran \\ Received 04 August 2021; received in revised form 04 November 2020; accepted 08 February 2021
}

DOI: https://doi.org/10.46604/ijeti.2021.6221

\begin{abstract}
The objective of this paper is to investigate the thermal behaviour and loadability characteristic of a yokeless and segmented armature axial-flux permanent-magnet (YASA-AFPM) generator, which uses an improved 3-D coupled electromagnetic-thermal approach. Firstly, a 1-kW YASA-AFPM generator is modelled and analysed by using the proposed approach; the transient and steady-state temperatures of different parts of the generator are determined. To improve the modelling accuracy, the information is exchanged between the thermal and electromagnetic models at each step of the co-simulation, considering both the accurate calculation of losses and the impacts of temperature rise on the temperature-dependent characteristics of the materials. Then, by using the proposed approach, the impact of the slot opening width and the turn number of stator segments on the generator loadability are investigated. After that, the experimental tests are performed. The results reveal the effectiveness and accuracy of the approach to predict the machine loadability and thermal behavior.
\end{abstract}

Keywords: permanent-magnet machines, finite element method, thermal analysis, loadability

\section{Introduction}

Over recent years, much research has been dedicated to the modeling, optimum design, and analysis of direct-drive AFPM machines. Using such electric machines has many benefits, such as a reduced number of the turbine drive-train components, reduced costs, increased system reliability, increased torque density, and improved efficiency. Due to having the competencies such as high power density, high efficiency, and low axial length, AFPM machine is a very good candidate to use as a direct-drive wind generator [1-3]. The AFPM generators could be designed in different topologies. The state of the art on topologies with a yokeless and segmented armature (YASA), which is an improved version of the double-sided AFPM topology, resulted in many positive aspects, such as short end coils, high torque density, low iron mass, and losses due to the deletion of unnecessary stator yoke, high slot filling factor, reduced mutual inductance, and fault tolerance [4].

To design a PM machine for a particular application, its thermal behavior must be investigated. Some concerns, such as the magnet stability, irreversible demagnetization, or any damage to the insulating materials, must be taken into consideration [5-6]. The winding temperature determines how long a machine can be loaded. Exceeding the thermal limits of the windings accelerates the oxidation of insulation materials, which in turn adversely affects their electrical properties and stability [7-8]. Thus, assessment and measurement of temperatures in the different parts of electric machines are of high importance.

* Corresponding author. E-mail address: s.jamali@yu.ac.ir

Tel.: +98-74-31005016; Fax: +98-74-31005000 
Analytical thermal modeling is an essential step in designing any electrical machinery to evaluate the temperature of different parts. The thermal analysis is beneficial in selecting machine materials. For example, thermal demagnetization risk is of great concern for permanent-magnet machines. Also, the heat tolerance of PMs and insulation materials is limited, and any violation of their permitted temperature rise makes them lose their efficiency and stability [9].

To investigate the thermal behavior of AFPM machines, distinct approaches, such as the computational fluid dynamics (CFD), lumped-parameter models, and finite element analysis (FEA), have been presented in the scientific literature [10-13]. The modeling of the machine losses as the heat sources requires an electromagnetic model. A coupled electromagnetic-thermal modeling technique was described for computing heat losses and evaluating temperature distribution in some electrical machines [14-15]. Furthermore, the thermal behavior of AFPM machines has been investigated through a 3-D thermal-magnetic finite-element analysis [16-17]. Nonetheless, in most of the above-mentioned approaches, the power losses, which play a basic role in the thermal analysis, have not been accurately calculated. For example, a general approximate relationship has been used to calculate iron losses. In addition, these studies overlook the fact that the effect of temperature rise on the properties of conductors and PMs is required for very accurate modeling, and it has not been included in the simulation process so far. The electrical, magnetic, and thermal properties of the materials, including the resistivity, magnetization, and thermal properties are function of the temperature. At each step of the simulation, variations in these properties affect the accuracy of thermal-magnetic analysis. Thus, in this study, to have accurate thermal modeling and analysis, considering the above-mentioned concerns, a new improved coupled electromagnetic-thermal analysis based on a 3-D magnetic and thermal co-simulation is presented and applied for the modeling and analysis of a YASA-AFPM generator. Another contribution of the paper is applying the approach to investigate the influence of the slot opening width and number of turns of each stator segment on the loadability of the studied YASA-AFPM generator. Finally, by using the conventional method, the proposed method, and the experimental tests, the loadability characteristics of the studied generator are obtained and compared.

The paper is structured as follows. In Section 2, the specifications of the studied YASA-AFPM machine are given. Then, in Section 3, the proposed thermal analysis approach is described. In Section 4, the results taken from the simulation and experimental tests (i.e. the loadability characteristic as well as the transient and steady-state temperatures of the coils and PMs) are discussed. Finally, the paper conclusions are provided in Section 5.

\section{Topology of the YASA Machine}

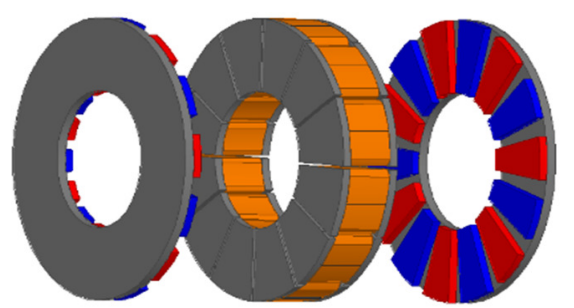

Fig. 1 The exploded view of the YASA-AFPM machine

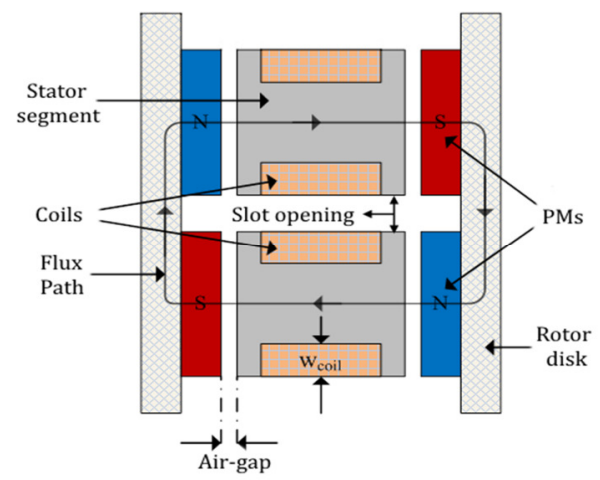

Fig. 2 The side view of the YASA-AFPM machine 
Table 1 The parameters and geometrical data of the studied YASA-AFPM generator

\begin{tabular}{|c|c|c|c|}
\hline Parameter & Value & Parameter & Value \\
\hline Rated Power $(\mathrm{W})$ & 1000 & Rated speed $(\mathrm{rpm})$ & 500 \\
\hline Outer diameter $(\mathrm{mm})$ & 180 & Inner diameter $(\mathrm{mm})$ & 87.65 \\
\hline Number of poles & 14 & Number of segments & 12 \\
\hline PM thickness $(\mathrm{mm})$ & 5 & Disk thickness $(\mathrm{mm})$ & 6.5 \\
\hline Air-gap length $(\mathrm{mm})$ & 1 & Slot opening width $(\mathrm{mm})$ & 2 \\
\hline Pole arc to pole pitch & 0.68 & Segment axial length $(\mathrm{mm})$ & 29.8 \\
\hline No-load phase voltage $(\mathrm{V})$ & 60 & Shoe axial length $(\mathrm{mm})$ & 5 \\
\hline Specific electrical loading $(\mathrm{A} / \mathrm{m})$ & 25000 & Width of coil $(\mathrm{mm})$ & 8.48 \\
\hline
\end{tabular}

The exploded view of the studied YASA-AFPM generator with 12 stator segments and 14 PM poles is shown in Fig. 1. Also, the side view of the YASA-AFPM machine is shown in Fig. 2. The main parameters and geometrical data of the studied YASA-AFPM generator are listed in Table 1 [18].

\section{Proposed Thermal Analyses}

In the most recent coupled thermal-magnetic analysis, losses have been estimated by using general methods. Also, in the previous studies, the effect of temperature change on some material properties, such as thermal conductivity and specific heat capacity, has not been considered. A much more accurate study will identify how the coupling interacts with other variables that are believed to be linked to material properties. In the typical case, lower iterations are conducted; it should be mentioned that the electromagnetic analysis implemented in the steady-state reduces time, while the transient operating mode that takes a great deal of time is solved. While various methods to conduct the thermal analysis of electrical machines are not linked with electromagnetic analysis during the simulation, it is vital to simulate magnetic and thermal model simultaneously since the both are dependent on each other [14-16].

In this study, two subsequent electromagnetic and thermal analyses are coupled, considering the effect of temperature changes on the thermal properties of the materials used in the generator structure. Here, to implement a highly accurate coupled electromagnetic-thermal approach during the simulation process, the temperature-dependent properties of the materials are considered as functions of the temperature and updated with the new calculated temperatures. The temperature-dependent thermal properties of materials, such as permanent magnets, copper coils, steel sheets, and soft iron, are included in the model. The magnetic analysis makes it possible to calculate the power losses by the Joule effect in the parts to be heated, and the governing equation used in the magnetic model is expressed as:

$$
\nabla \times\left(V_{0}\left[V_{r}\right] \nabla \times \vec{A}\right)+[\sigma]\left(\frac{\partial \vec{A}}{\partial t}\right)+\nabla V=0
$$

where $V_{0}, \mathrm{~V}_{\mathrm{r}}, A, \sigma$, and $V$ are the tensor of vacuum reluctivity, medium reluctivity, magnetic vector complex potential, conductivity of the medium, and electric scalar potential, respectively.

The copper, iron, mechanical, and PMs eddy current losses are the most significant power losses that occur in the AFPM machines. The mechanical losses include windage losses (air-solid friction) and bearing friction losses (solid-solid friction). Due to the low rotational speed of the studied YASA-AFPM generator, the mechanical losses are neglected. For the studied generator, which uses the relationships given in [18], the total mechanical losses are estimated as about 3.68 watts. In the proposed thermal analysis, at each step of the co-simulation, the losses created in the coils and stator segments, and the rotor disks are calculated by using the 3-D electromagnetic analysis, while at each step of the co-simulation, the temperature-dependent properties of the materials are updated with the temperature variations. 
The magnetization curve of the iron materials (grain-oriented steel sheets of the stator segments and soft iron used in the rotor disks), as well as the nonlinear demagnetization curve of the PM materials, are included in the analysis. The iron losses in the stator segments and in the rotor disks can be accurately calculated by using the following relation:

$$
d p_{F e}=k_{h}\left(f, B_{m}\right) f B_{m}^{2}+k_{e}\left(f, B_{m}\right) d^{2}\left(B_{m}\right)^{2}
$$

where $d$ is the derivative operator, $p_{F e}$ is the iron losses density, $B_{\mathrm{m}}$ is the peak value of the alternating flux density, $f$ is the frequency, $k_{e}\left(f, B_{\mathrm{m}}\right)$ and $k_{h}\left(f, B_{\mathrm{m}}\right)$ are the variable coefficients of the eddy currents and hysteresis losses, respectively [19].

Thus, in comparison to the conventional methods, which use constant coefficients for calculating total iron losses, in this study, the coefficients are fitted as a nonlinear function of $B_{\mathrm{m}}$ and $f$ according to the core loss curve of the used materials. For example, the core loss curve of the grain-oriented steel sheets (M4-Goes) used for the stator segments (at a frequency of 50 \& $60 \mathrm{~Hz}$ ) is shown in Fig. 3. After we calculate the average power dissipation in the iron volume regions by integrating the instantaneous losses density during a period, the resulted mean losses are given in the thermal analysis.

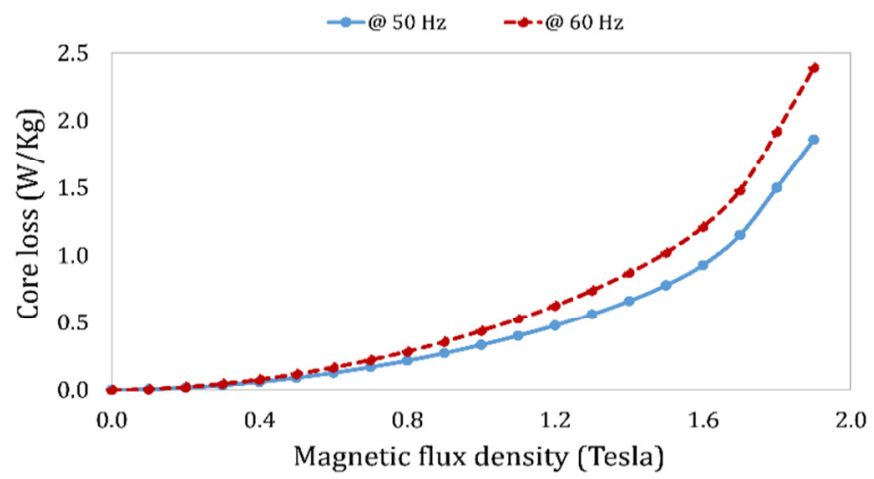

Fig. 3 The core loss curves of the M4-Goes

Due to the induced eddy currents in the PMs, the current density at the cross-section of the PMs can be calculated as follows:

$$
J_{m}=-\frac{1}{\rho_{m}} \frac{\partial \vec{A}}{\partial t}+J_{c}
$$

where $\rho_{m}$ and $J_{c}$ are the resistivity of the PM material and the constraint current density, respectively [20]. By integrating $\rho J_{m}{ }^{2}$ over the PM volume regions, the average PM losses are obtained.

Table 2 Variation of the copper properties with temperature [21-22]

\begin{tabular}{|c|c|c|}
\hline Specific Heat Capacity $(\mathrm{J} / \mathrm{kg} . \mathrm{K})$ & Thermal Conductivity $(\mathrm{W} / \mathrm{m} \cdot \mathrm{K})$ & Temperature $\left({ }^{\circ} \mathrm{C}\right)$ \\
\hline 384.70 & 400.73 & 20 \\
\hline 386.07 & 400.02 & 30 \\
\hline 387.42 & 399.31 & 40 \\
\hline 388.76 & 398.60 & 50 \\
\hline 390.07 & 397.88 & 60 \\
\hline 391.36 & 397.17 & 70 \\
\hline 392.63 & 396.46 & 80 \\
\hline 393.88 & 395.75 & 90 \\
\hline 395.12 & 395.05 & 100 \\
\hline
\end{tabular}

As the temperature changes, the electrical, magnetic, and thermal properties of the materials, including the resistivity, magnetization, specific heat capacity, and thermal conductivity, changes too. In order to calculate losses more accurately and to deal with the dependence of the material properties on temperature as with conductors, the specific heat capacity and thermal conductivity of the materials are considered in the proposed model as an exponential function of temperature as follows: 


$$
f(T)=a+b e^{\frac{T-T_{0}}{\tau}}
$$

where $a$ and $b$ are constant factors, $T_{0}$ is the ambient temperature, and $\tau$ is the temperature constant. Table 2 shows the data related to the copper.

To study the impact of the losses on the thermal behavior of the machine, the temperature evolution of the machine is investigated via the transient thermal analysis. The transient thermal behavior of the proposed model is simulated by using the following equation:

$$
\rho C_{p} \frac{\partial T}{\partial t}+\nabla \times(-k \nabla T)=q
$$

where $\rho C_{p}, k$ and $q$ are the volumetric heat capacity, thermal conductivity tensor, and volumetric power density of the heat sources, respectively.

The heat generated due to the power losses is transmitted through three heat transfer mechanisms, namely conduction, radiation, and convection. As it is widely accepted, convection is the most important mechanism, although radiation has been proven relevant in some cases. The heat generated due to the losses is transmitted through conduction in the solid parts of the electric machines, and through the convection in the fluids. In an AFPM machine with a single inner stator and two outer rotors, the heat generated in the coils is transferred to the stator via conduction and then is conducted to the machine's frame, where the frame surface is cooled by the environment. The rotor disks have no heat source, and the heat transfer is done via convection through the heated air movement in the air-gap. Because of the lamination, the temperature gradient over the stator area is significant. However, the high conductivity of the metal and the small size of the rotor cause the rotor area to have an insignificant temperature gradient. In the steady-state rotation, the rotor heating is uniform unless there are juts on the surfaces of the rotor, and they are exposed to more heated airflow. Obviously, the temperature increases from the inner radius towards the rotating clearance outlet, which is due to the air heating in the clearance. The maximum temperature occurs in the stator areas adjacent to the coils, especially in the areas closer to the inner radius owing to the higher amount of winding per volume [23]. The temperatures in the magnets are significantly lower than those of the stator segments because the PMs are directly mounted on the efficiently-convection-cooled rotor disks [24].

The conduction and radiation mainly depend on the geometry and physical properties, while the convection modeling requires fluid dynamics. Although the contribution of radiation to heat transfer is typically neglected in the thermal model, radiation coefficients are defined for each boundary of the presented model. The emissivity at the interface between rotor and shaft as well as the stator boundary is defined as $0.9 \mathrm{~W} /\left(\mathrm{m}^{2} \cdot{ }^{\circ} \mathrm{C}^{4}\right)$. The rotor disk is a freely rotating disk, i.e. a disk that freely rotates around its center. For such a disk, the coefficients of the convection heat transfer in the laminar and turbulent flow regimes have been expressed as [25-26]:

$$
\bar{h}=\frac{k}{R} \times \overline{N u}
$$

where $R$ is the radius of the disk, and the average Nusselt number $(\overline{N u})$ is obtained according to the different flow conditions. The critical Reynold number for transition is:

$$
r_{c}=\left(2.5 \times 10^{5} \frac{v}{\Omega}\right)^{\frac{1}{2}}
$$

where $\Omega$ is the angular speed, and $v$ is the air dynamic viscosity. As $r_{c}>R$, the air flowing in the air-gap is laminar. The Nusselt number to heat transfer coefficient of the outside rotor disk can be considered as: 


$$
\overline{N u}=\frac{2}{5} \operatorname{Re}^{2}\left(1+G r / R e^{2}\right)^{1 / 4}
$$

where Reynolds $(R e)$ and Grashof $(G r)$ numbers are obtained by:

$$
\begin{aligned}
& R e=\frac{2 \pi n \rho R_{\text {out }}^{2}}{\mu} \\
& G r=\frac{\beta g R^{3} \pi^{\frac{3}{2}} \Delta T}{v^{2}}
\end{aligned}
$$

where $\beta$ is the coefficient of thermal expansion, $\Delta T$ is the temperature difference between the disk surface and surrounding air, $\rho$ is the specific density of the cooling medium, $n$ is the rotational speed, and $\mu$ is the dynamic viscosity of the fluid.

Regarding peripheral edge of rotor, the Nusselt number for average heat transfer coefficient around the radial periphery can be written as:

$$
\overline{N u}=0.133 \operatorname{Re}^{\frac{2}{3}} p r^{\frac{1}{3}}
$$

where $p r$ is the Prandtl number. The value of Nusselt on rotating shaft can be expressed as:

$$
\overline{N u}=0.119(R e)^{\frac{2}{3}}
$$

As for the calculation of the heat transfer coefficient in the rotor-stator system, the Nusselt number is:

$$
\overline{N u}=0.333 Q /(\pi \nu R)
$$

The volumetric flow rate of the machine (Q) at rated speed (500 rpm) is considered as $0.0025 \mathrm{~m}^{3} / \mathrm{s}$ according to the measured characteristic curves of the machine. The assumptions are based on the natural air-cooling.

The process of the new multi-physics 3D FE magnetic-thermal analysis is characterized based on the step-by-step flowchart illustrated in Fig. 4. At the beginning of the analysis, as defined in Equation (2), the core loss which depends on the flux density and frequency, is calculated for the iron parts of the machine. By fitting the core loss curve of the used material (refer to Fig. 3) as a nonlinear function of flux density $\left(B_{m}\right)$ and frequency $(f)$, the coefficients are determined. The coefficients that fit a set of data in a least-squares sense are computed by using the Curve Fitting Toolbox in MATLAB. Then, the coefficients are added to the 3D-FEM magnetic model. All types of losses are calculated by using 3D transient EM analysis based on the initial (ambient) temperature, and the 3D thermal analysis simulation with FEA uses the estimated losses as the heat sources. The boundary conditions and the heat transfer coefficients are defined; the amount of the generated heat in all regions is obtained. The flow of heat is transferred through the materials.

Parameters, such as thermal conductivity (k) and specific heat capacity $\left(C_{p} T\right)$, are temperature-dependent and can strongly influence the thermal analysis. Therefore, the accurate estimation of these parameters is necessary in case of the transient analysis. As shown in Fig. 4, an iteration-based method is used to determine the exact amount of the thermal variables $\left(\mathrm{k} \& \mathrm{C}_{p} \mathrm{~T}\right)$ that will be used in the thermal analysis. In the first iteration at the beginning of the analysis, two initial values are assumed for the thermal conductivity and the specific heat capacity. In other words, the thermal variables of the materials in different regions of the model take on initial values (initialization step). Then, 3D finite element thermal analysis is applied. In the next step, post-processing is performed, and temperatures of the different regions of the model are estimated. For each 
region of the model, the thermal variables at the new temperature $\left(\mathrm{k}_{\text {interp }} \& \mathrm{C}_{p} \mathrm{~T}_{\text {interp }}\right)$ are calculated by using the interpolation function (based on Table 2). Then, considering the previous values of the thermal variables, two separate error criteria $\left(\varepsilon_{\mathrm{k}} \&\right.$ $\varepsilon_{\mathrm{C}_{p} \mathrm{~T}}$ ) are estimated as follows:

$$
\begin{aligned}
& \varepsilon_{\mathrm{k}}=\left|\mathrm{k}_{\text {interp }}-\mathrm{k}\right| / \mathrm{k}_{\text {interp }} \\
& \varepsilon_{\mathrm{C}_{p} \mathrm{~T}}=\left|\mathrm{C}_{p} \mathrm{~T}_{\text {interp }}-\mathrm{C}_{p} \mathrm{~T}\right| / \mathrm{C}_{p} \mathrm{~T}_{\text {interp }}
\end{aligned}
$$

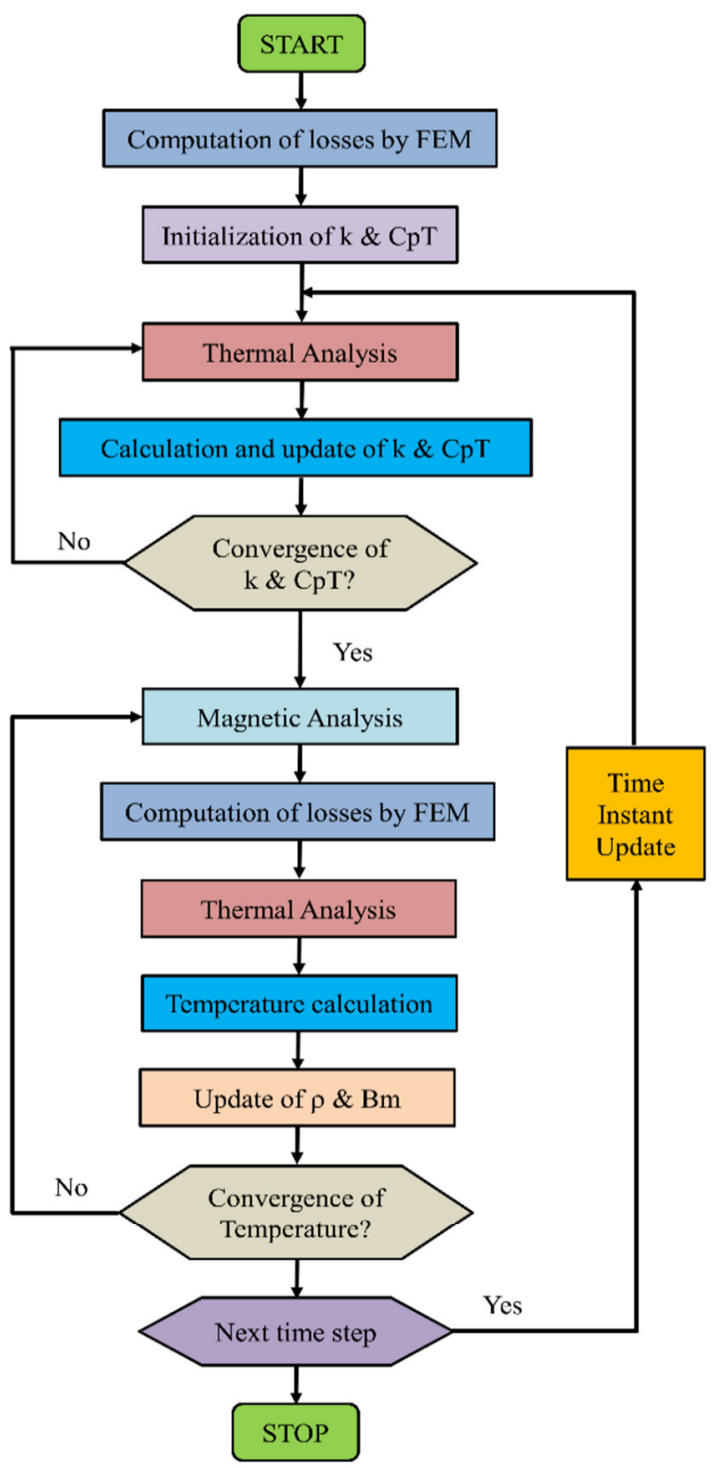

Fig. 4 The flowchart of the proposed 3-D coupled thermal-magnetic analyses

As observed in Fig. 4, the flowchart has an update block for the thermal variables $\left(\mathrm{k} \rightarrow \mathrm{k}_{\mathrm{upd}} \& \mathrm{C}_{p} \mathrm{~T} \rightarrow \mathrm{C}_{p} \mathrm{~T}_{\mathrm{upd}}\right)$. The operation of the update block is based on the algorithm described below; for example, updating $\mathrm{C}_{p} \mathrm{~T}$ is done according to the flowchart shown in Fig. 5. A similar procedure is used to update $\mathrm{k}$. The criterion to modify (update) the values of $\mathrm{k}$ and $\mathrm{C}_{p} \mathrm{~T}$ which is in relations 14 and 15 is the value of the error defined for each of these variables $\left(\varepsilon_{\mathrm{k}} \& \varepsilon_{\mathrm{C}_{p} \mathrm{~T}}\right)$. In each iteration, each thermal variable is modified (updated) according to its own error. As shown in Fig. 5, if the value of the error associated with each thermal variable is less than or equal to the value of $\xi(\varepsilon \leq \xi)$, the updated value of that variable is considered equal to its previous value $\left(\mathrm{k}_{\mathrm{upd}}=\mathrm{k} \& \mathrm{C}_{p} \mathrm{~T}_{\mathrm{upd}}=\mathrm{C}_{p} \mathrm{~T}\right)$. Otherwise, the interpolated values of the thermal parameters $\left(\mathrm{k}_{\text {interp }}=\mathrm{C}_{p} \mathrm{~T}_{\text {interp }}\right)$ are compared to their previous values. For instance, 


$$
\begin{cases}\mathrm{C}_{p} \mathrm{~T}_{\text {upd }}=\mathrm{C}_{p} \mathrm{~T}-\varepsilon_{\mathrm{C}_{p} \mathrm{~T}} \frac{\mathrm{C}_{p} \mathrm{~T}}{2} & \mathrm{C}_{p} \mathrm{~T}>\mathrm{C}_{p} \mathrm{~T}_{\text {interp }} \\ \mathrm{C}_{p} \mathrm{~T}_{\text {upd }}=\mathrm{C}_{p} \mathrm{~T}+\varepsilon_{\mathrm{C}_{p} \mathrm{~T}} \frac{\mathrm{C}_{p} \mathrm{~T}}{2} & \mathrm{C}_{p} \mathrm{~T}>\mathrm{C}_{p} \mathrm{~T}_{\text {interp }}\end{cases}
$$

The thermal variables will keep being updated until $\varepsilon \leq \xi$. In this study, a limit iteration discrepancy of $0.5 \%$ is adopted $(\xi=0.5 \%)$.

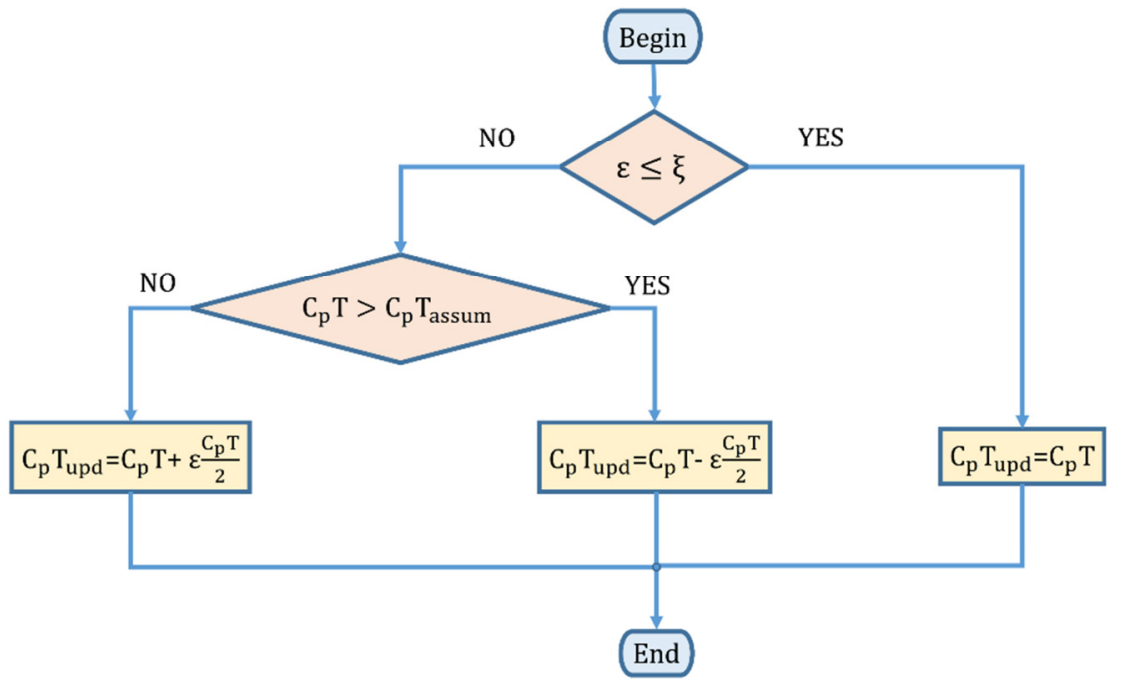

Fig. 5 The used flowchart to update the specific heat capacity

After updating the thermal conductivity and the specific heat capacity of the material, the coupled electromagnetic-thermal analysis is performed. As mentioned earlier, in the electromagnetic analysis, some parameters, such as the resistivity of conductors and the residual flux density of permanent magnets, are temperature-dependent. In addition, the losses calculated via the electromagnetic analysis are the inputs of the thermal analysis. Therefore, the interaction between the two analyses should be taken into account. Due to the interaction between the thermal and magnetic analyses, it is better to exchange data between the two analyses during the simulation. The co-simulation provides data exchanges between the thermal and magnetic analyses. In the second loop of the flowchart in Fig. 4, the data exchange continues until the temperature field convergence condition is met. At each time step, the convergence of the temperature field is checked by using Equation (16), which is based on newly imported and previously imported temperature fields.

$$
\sqrt{\frac{\sum_{j}\left(T_{j, \text { mew }} T_{j, \text { old }}\right)^{2}}{\sum_{j}\left(T_{j, \text { mew }}\right)^{2}}} \leq \xi_{T}
$$

where $T$ is the temperature, $j$ is the node number, and $\xi_{T}$ is the desired accuracy.

The steps taken during the coupled electromagnetic-thermal analysis in the second loop of the flowchart (Fig. 4) are given in Table 3. This multi-physics procedure is implemented by using Altair Flux - MATLAB-Simulink coupling as presented in Table 3. This coupling realizes the solving of magnetic and thermal transient equations at each time step. First, the electromagnetic aspect is analyzed and then, the thermal one. Once the convergence of multi-physics coupled procedure is achieved at each moment, the calculations of the next time step will be performed.

The coupling process includes the following steps:

(1) Preparation of the Altair Flux project: the physical application (magnetic, electric or thermal), geometry, mesh, physics specific description (definition of materials and their characteristics), and the multi-physics input and output parameters are defined. Also, the face and volume regions are assigned. 
(2) Generation of the "component file" via the Altair Flux: the coupling "component file" is necessary in order to transfer information from the Altair Flux project to the MATLAB-Simulink. The component file has an extension in ".F2MS" format. By using the 3D Altair Flux software, two component files are created. One is for the electromagnetic model, and the other is for the thermal model (in the transient application).

(3) Creation of MATLAB-Simulink model: in MATLAB-Simulink, two blocks are created called coupling blocks, and these blocks are addressed by these two component files. In addition to the Altair Flux-Simulink coupling library, other libraries are added to be used to update the temperature-dependent properties of the materials in the time steps where the temperature changes.

(4) Configuration of the Simulink model parameters: an attempt has been made to choose an optimal solver by making a compromise between accuracy and simulation time. Fixed-step solver which implements the ODE3 method is selected to solve the model. Fixed-step size or fundamental sample time is considered as $25 \mathrm{E}-5$ seconds.

(5) Launching the simulation.

(6) Post-processing of results.

Table 3 The steps taken during the second loop of the flowchart in Fig. 4

\begin{tabular}{|c|c|c|c|}
\hline Step & Magnetic & Thermal & Software \\
\hline 1 & $\begin{array}{l}\text { Performing transient magnetic analysis at the } \\
\text { current time step } t_{i} \text {, and calculation of losses }\end{array}$ & - & Altair Flux \\
\hline \multirow{2}{*}{2} & Exporting losses & - & \\
\hline & - & Importing losses. & \\
\hline 3 & - & $\begin{array}{l}\text { Performing transient thermal analysis } \\
\text { at the time step } \mathrm{t}_{\mathrm{i}} \text {, and obtaining the } \\
\text { temperature field }\end{array}$ & Altair Flux \\
\hline \multirow{2}{*}{4} & - & Exporting the temperature field & \\
\hline & Importing the temperature field & - & \\
\hline 5 & $\begin{array}{l}\text { Updating some of the temperature-dependent } \\
\text { parameters in the electromagnetic model, such as } \\
\text { the resistivity of conductors and the residual flux } \\
\text { density of PMs }\end{array}$ & - & MATLAB-Simulink \\
\hline 6 & \multicolumn{2}{|c|}{ Checking the convergence of the temperature field at the time step $t_{i}$, using Eq. (17) } & MATLAB-Simulink \\
\hline 7 & $\begin{array}{l}\text { If the temperature field convergence is met, go to } \\
\text { the next time step } t_{i+1} \text {; otherwise, return to Step } 1\end{array}$ & & \\
\hline
\end{tabular}

The numerical solution of the equations employs a 3-D finite volume discretization approach in which the software package Altair Flux is used for the thermal and magnetic analysis. The following assumptions are considered in the 3-D model: i) the heat generated per unit volume is uniformly distributed throughout the different parts; ii) the heat transferred by the radiation is neglected; iii) the ambient temperature is taken as the measured one $\left(28.1^{\circ} \mathrm{C}\right)$.

Owing to the symmetry, half of the machine is used in the coupling analysis, and a high-quality mesh is applied to produce results with an acceptable level of accuracy. Necessarily, the magnetic and thermal models do not have the same mesh. The estimated losses from the 3D electromagnetic analysis are used as the heat sources in 3D thermal simulation. For a more accurate estimation of losses, the accuracy of the electromagnetic analysis is of great importance. For this purpose, in the electromagnetic model, finer meshes are used for the air-gap and nearby areas, whereas an auto-adapting mesh is used for the other areas. In the thermal model, finer meshes are used in the regions where the temperature changes, and heat flux vectors are expected to rapidly change their value and direction. If the meshes of the regions are different for electromagnetic and thermal models, Multi-point Support is implemented in Altair Flux software. This is necessary for data exchange if two regions have different meshes. The coordinates of the nodes are transferred from one to another via Multi-point Support. The meshing used in the thermal analysis is illustrated in Fig. 6. The detailed data related to the meshing are given in Table 4. 


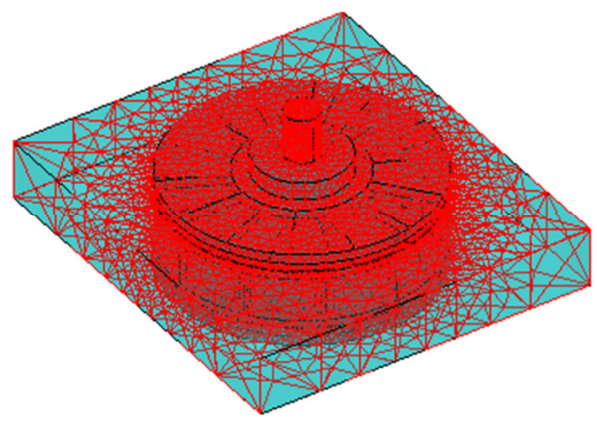

(a) $3 \mathrm{D}$ view

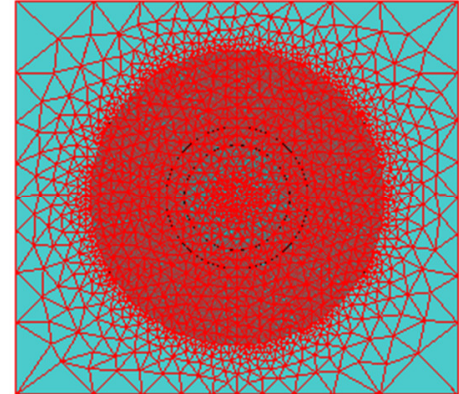

(b) $2 \mathrm{D}$ view

Fig. 6 The meshing used in the thermal analysis

Table 4 The detailed data related to the meshing used in the electromagnetic and thermal analyses

\begin{tabular}{|c|c|c|c|}
\hline \multicolumn{2}{|c|}{ Thermal analysis } & \multicolumn{2}{c|}{ Electromagnetic analysis } \\
\hline Number of nodes & 83181 & Number of nodes & 143598 \\
\hline Number of line elements & 9942 & Number of line elements & 10969 \\
\hline Number of surface elements & 169378 & Number of surface elements & 167120 \\
\hline Number of volume elements & 460031 & Number of volume elements & 842827 \\
\hline Mesh order & 2nd order & Mesh order & 2nd order \\
\hline
\end{tabular}

Although a smaller time step increases accuracy, it also increases the simulation time. Solving the coupled multiphysics problems may take a long computational time (time step: 25E-5 seconds; computation time: 488 minutes). Even if the computation time is relatively higher than other approaches and the calculating process becomes more complex, high-performance computing (HPC) technology for the analysis can address the problem to consume less time and easier to be carried out.

Based on the optimized dimensions, the YASA-AFPM generator is fabricated from the main materials including copper (for stator coils), grain-oriented steel sheets (M4-Goes) for the stator segments, white Teflon sheet, epoxy resins, soft iron (ST37) for the rotor disks, and permanent magnets (N35) [18]. The thermal class of the insulations is B, which tolerates a maximum hot spot temperature of $130^{\circ} \mathrm{C}$.

\section{Results}

\subsection{Simulation results}

In this section, by using a number of the proposed 3-D coupled magnetic-thermal FEA simulations, the impacts of the slot opening width and the number of turns wound around each stator segment on the loadability characteristic of the studied YASA-AFPM generator are investigated, and the obtained results are given in Fig. 7 and Fig. 8, respectively. The loadability of a generator can be defined as the maximum power that the generator can deliver over the output current range from zero to the rated current. In addition, the generator loadability characteristic shows the changes in the generator output power versus the load current. To obtain the loadability characteristics, considering different loads for the generator, including $100 \mathrm{ohms,} 30$ ohms, $15 \mathrm{ohms}$, $10 \mathrm{ohms}, 8 \mathrm{ohms}, 6 \mathrm{ohms}$, etc., a separate simulation is performed for each load. For each simulation (related to each load), the initial temperature is assumed to be equal to the ambient temperature $\left(25^{\circ} \mathrm{C}\right)$.

As shown in Fig. 7, a decrease in the slot opening width has resulted in a decrease in the YASA-AFPM generator loadability. The reason for this phenomenon can be explained as following. By reducing the slot opening width, due to the increased area of the stator segments seen from the air-gap side, the cross-section area of the armature reaction flux increases, which in turn reduces the armature reaction reluctance and thus increases the generator synchronous reactance. Given that the output power of the synchronous generators is inversely related to the synchronous reactance, so it can be concluded that decreasing the slot opening width leads to a decrease in the generator loadability. 
Therefore, when designing the YASA-AFPM generators, it is important to consider a reasonable value for the slot opening width. This is due to the fact that although increasing the slot opening width leads to better loadability, it also leads to increased cogging torque, which has an adverse effect on the generator performance. Accordingly, to choose an appropriate width for the slot opening, it is better to make a compromise between the generator loadability and its cogging torque. It should be noted that the discussion about cogging torque is beyond the scope of this paper.

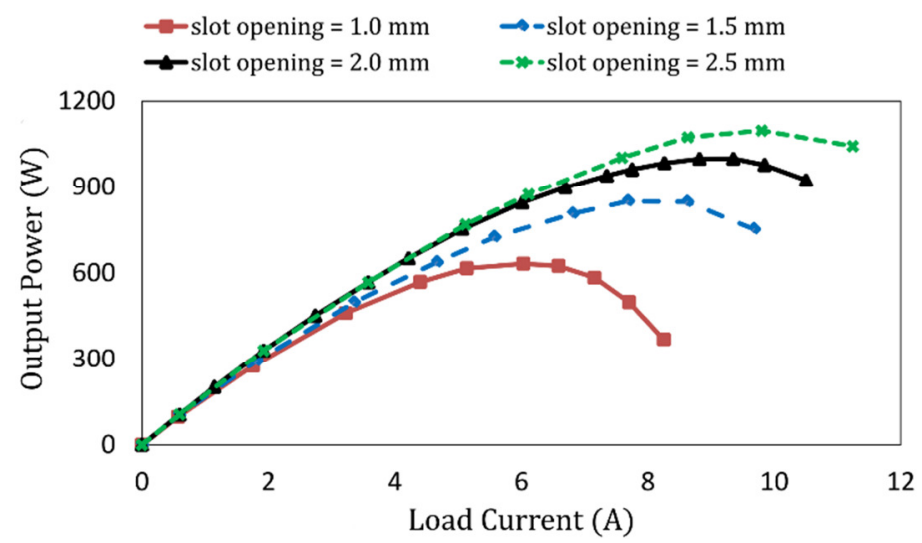

Fig. 7 The impact of the slot opening width on the YASA-AFPM generator loadability

Also, as shown in Fig. 8, the impact of the number of turns wound around each of the stator segments on the YASA-AFPM generator loadability characteristic is investigated. It can be seen that the turn's number does not affect the maximum output power of the generator, but for a lower turn number, a design with a higher output current and a lower output voltage can be achieved.

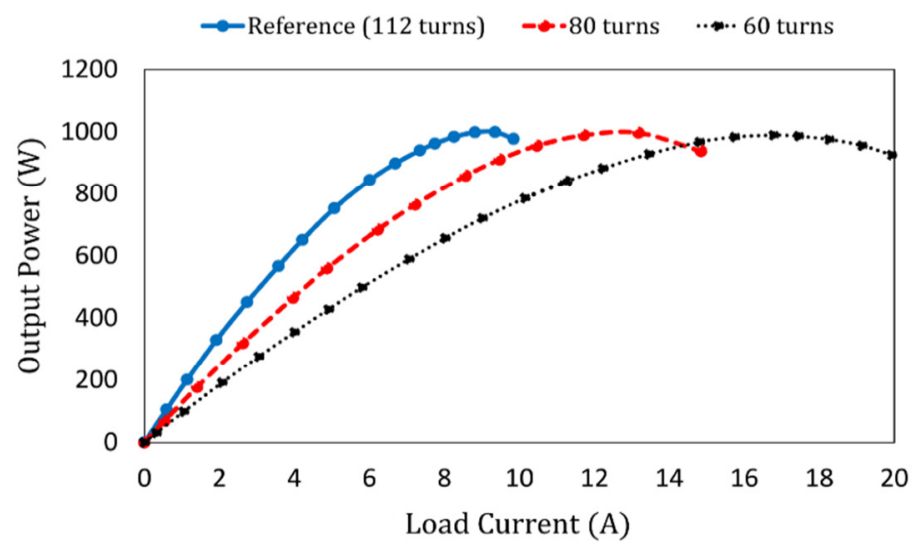

Fig. 8 The impact of the turns number of each stator segment on the YASA-AFPM generator loadability

The fact that changing the stator turn's number does not affect the maximum output power of the generator can be explained as follows. By changing the armature turn's number, the generator excitation voltage changes proportionally. Besides, as the generator is operated in off-grid mode, the terminal voltage of the generator also changes approximately in proportion to the stator turn's number. In the PM generators, the synchronous reactance is directly related to the square of the stator turn's number [26]. Given that the maximum output power of a synchronous generator is directly related to the product of the excitation voltage and the terminal voltage but is inversely related to the synchronous reactance, it is expected that changing the stator turn's number does not affect the maximum output power of the generator.

\subsection{Experimental results}

The structure of the stator segments as well as the rotor disks of the studied YASA-AFPM generator are illustrated in Fig. 9. The core of the stator segments is made of the grain-oriented electrical steel sheets (M4-Goes), and a concentrated coil of 112 turns is wound around each of the stator segments. The rotor disks are made of soft iron (ST37), and 14 PM poles are 
mounted on each rotor disk. To reduce the PM eddy losses, each magnet pole is divided into 3 pieces. To validate the proposed analysis, as shown in Fig. 10, an experimental setup is developed; the prototype of the studied YASA-AFPM generator is driven by a DC motor at nominal speed (500 rpm) and loaded with different resistive loads.

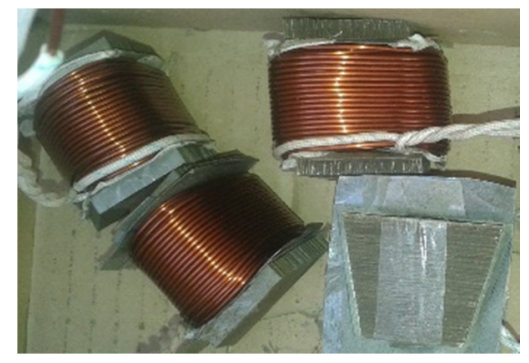

(a) Stator segments

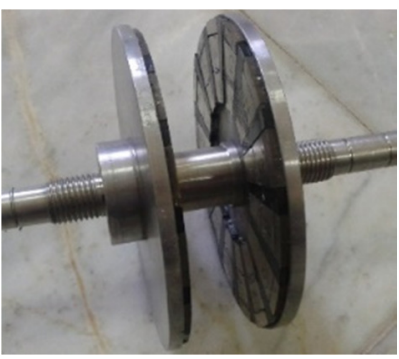

(b) Rotor disks

Fig. 9 The prototype of the YASA-AFPM generator

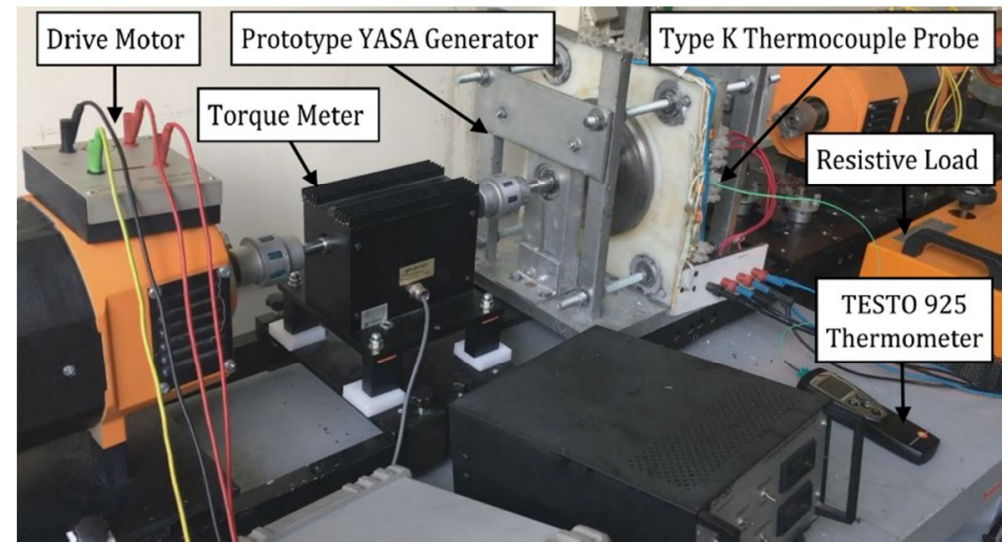

Fig. 10 Experimental setup

Fig. 11 shows the loadability characteristic of the studied YASA-AFPM generator, which is obtained via the experimental tests, conventional and proposed 3-D coupled magnetic-thermal FEA simulations. It should be mentioned that in the conventional 3-D coupled magnetic-thermal method, the thermal properties of materials do not change with the temperature variations.

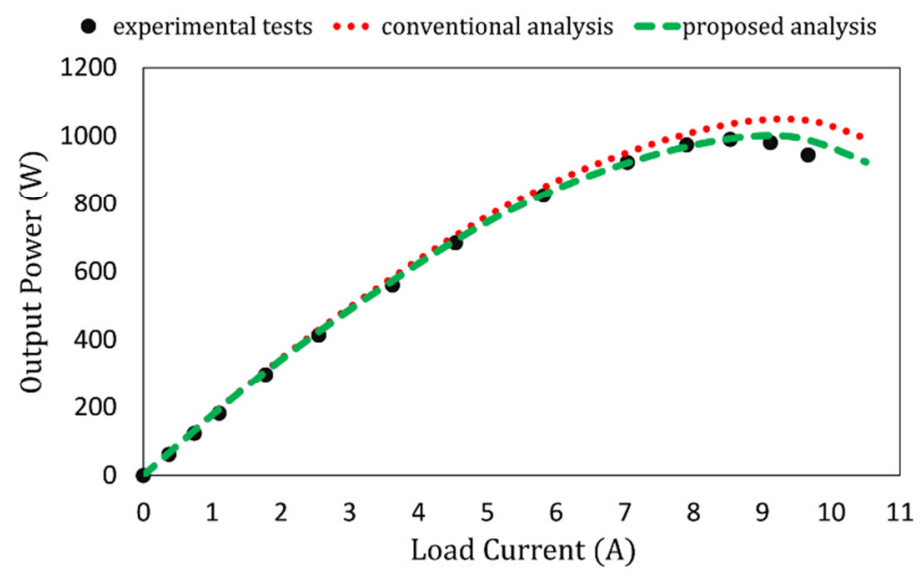

Fig. 11 The loadability characteristic obtained via the experimental tests, conventional and proposed 3-D coupled FEA

According to Fig. 11, the experimental results show a good agreement with those obtained via the proposed 3-D coupled magnetic-thermal FEA simulations. In addition, it can be seen that the results obtained from the proposed and conventional methods are somewhat different. The loadability characteristic obtained through the proposed method is somewhat lower than the one obtained through the conventional approach, especially for the higher load currents. For the loadability characteristics obtained via the conventional and proposed approaches and for the load currents up to 10 amps, the maximum error regarding 
the experimental loadability characteristic is calculated as about $7.51 \%$ and $4.09 \%$, respectively. The justification for this is as follows. In the proposed 3-D coupled magnetic-thermal method, the effects of the temperature changes on the material properties are included in each step of the simulation, which successively influences the electromagnetic analyses. Thus, the proposed coupled analyses can be applied for an accurate examination of the electrical machines' performance. For the load currents above 8.5 amps, a further increase in the load current results in a decrease in the YASA generator output power. This is because of a redundant increase in the armature reaction effect, which in turn leads to the demagnetization of PMs and thus decreases the air-gap magnetic flux density.

At the nominal rotational speed $(500 \mathrm{rpm})$ and full-load condition, with the natural air-cooling system, a TESTO 925 Type K thermocouple thermometer and a TESTO 830-T1 infrared thermometer are used to measure the temperature of coils and PM surface, respectively.

For the nominal output current $(8 \mathrm{amps})$, where the generator output power is the nominal power, the transient temperatures of the studied YASA-AFPM generator obtained via the conventional and proposed 3-D coupled magnetic-thermal FEA simulations and the experimental tests are compared in Fig. 12.

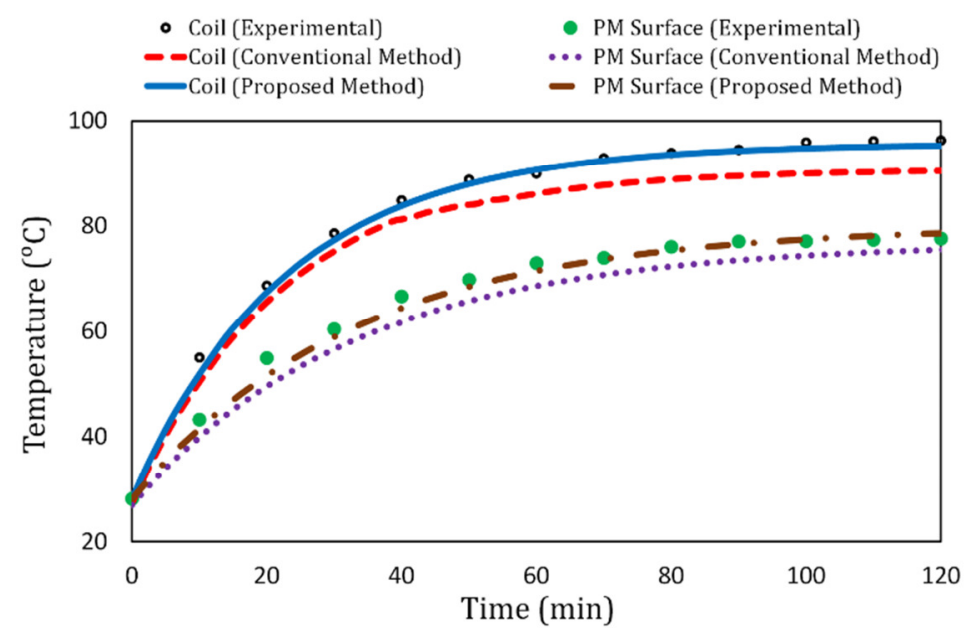

Fig. 12 Temperatures obtained in the coil and PM surface under full-load conditions

As it can be seen from the curves, the results achieved from the 3-D coupled electromagnetic-thermal analysis are in good agreement with those achieved from the measurements, and this confirms that the proposed approach can execute the thermal analysis with acceptable accuracy.

In order to measure the temperature, the probe type $\mathrm{K}$ (maximum temperature measurement: $+400{ }^{\circ} \mathrm{C}$ ) is located within the coils as can be seen in the Experimental setup (The green-colored probe in Fig. 10). It should be mentioned that the maximum temperature of the generator is occurred in the coils and stator segments. Strictly speaking, thanks to the good thermal contact and the high thermal conductivity of both materials, there is a slight temperature difference between the stator segments and coils.

According to Fig. 12, the steady-state temperature of the coils is reached after 115 minutes. The steady-state temperature of the stator coils obtained by conventional, proposed, and experimental methods are approximately as $90.7{ }^{\circ} \mathrm{C}, 95.5^{\circ} \mathrm{C}$, and $96.4{ }^{\circ} \mathrm{C}$, respectively. Hence, regarding the experimental method, the error of conventional and proposed methods in estimating the steady-state temperature of the stator coils is evaluated as about $5.9 \%$ and $0.93 \%$, respectively. Also, by using the curves in Fig. 12, which show the temperature time variations of the stator coils, the thermal time constant of the coils for the conventional, proposed, and experimental methods are estimated as about 22.19 minutes, 22.63 minutes, and 22.58 minutes, respectively. The difference between the results of the proposed and conventional methods indicates the effects and high importance of accurate calculation of losses. The temperatures in the PMs are significantly lower than those of the coils and 
stator segments because the PMs are mounted on the surface of the rotor disks. The heat generated in the coils and the stator segments is axially dissipated through the convective flux from the stator segments to the air-gap, where the convective cooling occurs from the stator to the rotor. Despite the heat flux toward the rotor disks, a good convective cooling at the backplane of the disks can maintain the temperature of magnets within the allowable range. Fig. 13 illustrates the temperature distribution in different parts of the machine derived from the analyses under full load at $500 \mathrm{rpm}$ with a natural air-cooling system.

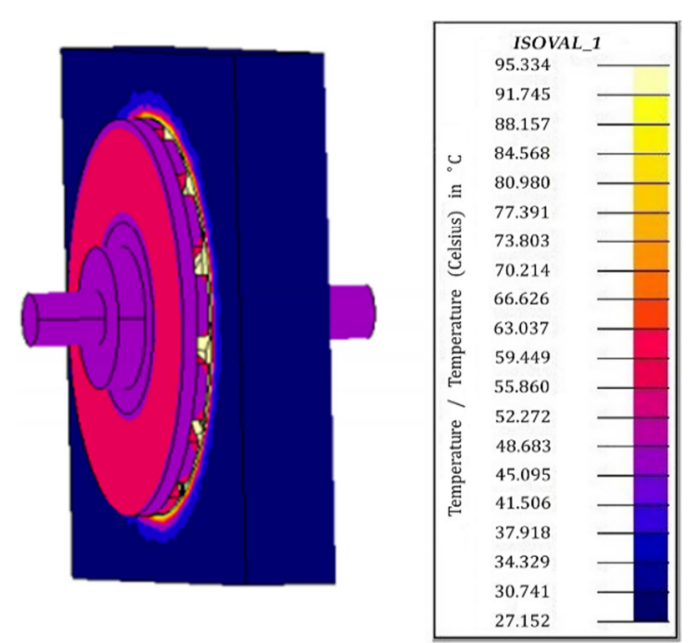

Fig. 13 The contour plots of the steady-state temperature distribution at full load

\section{Conclusions}

In this study, the loadability and thermal behavior of a $1 \mathrm{~kW}$ YASA-AFPM generator were investigated by using an improved coupled electromagnetic-thermal analysis. The impacts of the slot opening width and number of turns of each stator segment, on the YASA-AFPM generator loadability, were studied carefully. It presented that the generator loadability can be undesirably reduced if an inappropriate value is selected for the slot opening width. In addition, the temperature distribution obtained for the different parts of the generator showed that the design meets the generator thermal specifications under the full-load conditions. By doing the experimental tests on the prototype of the YASA-AFPM generator, the loadability characteristic and the temperature variations of the PMs and coils were obtained and compared with those obtained via the proposed and conventional coupled analysis. Comparing the simulation and experimental results revealed that the proposed approach has higher accuracy in the thermal and performance analysis than the conventional method. Thus, this analysis can be applied to accurately investigate the performance of electric generators. Studying the cooling system effect on the generator performance can still be a subject for further research.

\section{Conflicts of Interest}

The authors declare no conflict of interest.

\section{References}

[1] S. Kurian, T. K. Sindhu, and E. P. Cheriyan, "Review on Developments in Wind Energy Generation and its Integration to Utility Grid," International Review on Modelling and Simulations, vol. 6, no. 5, pp. 1523-1532, 2013.

[2] M. Cheng and Y. Zhu, "The State of the Art of Wind Energy Conversion Systems and Technologies: A Review," Energy Conversion and Management, vol. 88, pp. 332-347, December 2014.

[3] Y. Chen, P. Pillay, and A. Khan, "PM Wind Generator Topologies," IEEE Transactions on Industry Applications, vol. 41, no. 6, pp. 1619-1626, November 2005.

[4] B. Zhang, T. Seidler, R. Dierken, and M. Doppelbauer, "Development of a Yokeless and Segmented Armature Axial Flux Machine,” IEEE Transactions on Industrial Electronics, vol. 63, no. 4, pp. 2062-2071, April 2016. 
[5] A. Di Gerlando, G. M. Foglia, M. F. Iacchetti, and R. Perini, "Parasitic Currents in Stray Paths of Some Topologies of YASA AFPM Machines: Trend with Machine Size," IEEE Transactions on Industrial Electronics, vol. 63, no. 5, pp. 2746-2756, May 2016.

[6] M. A. Fakhfakh, M. H. Kasem, S. Tounsi, and R. Neji, “Thermal Analysis of a Permanent Magnet Synchronous Motor for Electric Vehicles,” Journal of Asian Electric Vehicles, vol. 6, no. 2, pp. 1145-1151, January 2008.

[7] H. Vansompel, P. Leijnen, and P. Sergeant, "Multiphysics Analysis of a Stator Construction Method in Yokeless and Segmented Armature Axial Flux PM Machines,” IEEE Transactions on Energy Conversion, vol. 34, no. 1, pp. 139-146, March 2019.

[8] A. Boglietti, A. Cavagnino, and D. Staton, "Determination of Critical Parameters in Electrical Machine Thermal Models," IEEE Transactions on Industry Applications, vol. 44, no. 4, pp. 1150-1159, July 2008.

[9] D. Joo, J. Cho, K. Woo, B. Kim and D. Kim, "Electromagnetic Field and Thermal Linked Analysis of Interior Permanent-Magnet Synchronous Motor for Agricultural Electric Vehicle,” IEEE Transactions on Magnetics, vol. 47, no. 10, pp. 4242-4245, October 2011.

[10] N. Rostami, M. R. Feyzi, J. Pyrhonen, A. Parviainen, and M. Niemela, "Lumped-Parameter Thermal Model for Axial Flux Permanent Magnet Machines,” IEEE Transactions on Magnetics, vol. 49, no. 3, pp. 1178-1184, March 2013.

[11] D. Staton, S. J. Pickering, and D. Lampard, "Recent Advancement in the Thermal Design of Electric Motors," SMMA Fall Technical Conference "Emerging Technologies for Electric Motion Industry”, October 2001 pp. 1-11.

[12] Y. Xu, M. Ai, and Y. Yang, "Heat Transfer Characteristic Research Based on Thermal Network Method in Submersible Motor," International Transactions on Electrical Energy Systems, vol. 28, no. 3, p. e2507, March 2018.

[13] A. Boglietti, A. Cavagnino, D. Staton, M. Shanel, M. Mueller, and C. Mejuto, "Evolution and Modern Approaches for Thermal Analysis of Electrical Machines," IEEE Transactions on Industrial Electronics, vol. 56, no. 3, pp. 871-882, March 2009.

[14] P. K. Vong and D. Rodger, "Coupled Electromagnetic-Thermal Modeling of Electrical Machines," IEEE Transactions on Magnetics, vol. 39, no. 3, pp. 1614-1617, May 2003.

[15] P. Holmberg and M. Leijon, "Coupled FEM and Lumped Circuit Model of the Electromagnetic Response of Coaxially Insulated Windings in Two Slot Cores,” European Transactions on Electrical Power, vol. 17, no. 6, pp. 554-568, November 2007.

[16] F. Marignetti, V. D. Colli, and Y. Coia, "Design of Axial Flux PM Synchronous Machines Through 3-D Coupled Electromagnetic Thermal and Fluid-Dynamical Finite-Element Analysis," IEEE Transactions on Industrial Electronics, vol. 55, no. 10, pp. 3591-3601, October 2008.

[17] H. Vansompel, A. Rasekh, A. Hemeida, J. Vierendeels, and P. Sergeant, "Coupled Electromagnetic and Thermal Analysis of an Axial Flux PM Machine," IEEE Transactions on Magnetics, vol. 51, no. 11, pp. 1-4, November 2015.

[18] S. J. Arand and M. Ardebili, "Multi-Objective Design and Prototyping of a Low Cogging Torque Axial-Flux PM Generator with Segmented Stator for Small-Scale Direct-Drive Wind Turbines," IET Electric Power Applications, vol. 10, no. 9, pp. 889-899, November 2016.

[19] D. M. Ionel, M. Popescu, M. I. McGilp, T. J. E. Miller, S. J. Dellinger, and R. J. Heideman, "Computation of Core Losses in Electrical Machines Using Improved Models for Laminated Steel,” IEEE Transactions on Industry Applications, vol. 43, no. 6, pp. 1554-1564, November 2007.

[20] D. Ishak, Z. Q. Zhu, and D. Howe, "Eddy-Current Loss in the Rotor Magnets of Permanent-Magnet Brushless Machines Having a Fractional Number of Slots Per Pole," IEEE Transactions on Magnetics, vol. 41, no. 9, pp. 2462-2469, September 2005.

[21] C. Y. Ho, R. W. Powell, and P. E. Liley, "Thermal Conductivity of the Elements," Journal of Physical and Chemical Reference Data, vol. 1, no. 2, pp. 279-421, 1972.

[22] G. K. White and S. J. Collocott, "Heat Capacity of Reference Materials: Cu and W," Journal of Physical and Chemical Reference Data, vol. 13, no. 4, pp. 1251-1257, October 1984.

[23] S. Kahourzade, A. Mahmoudi, A. Gandomkar, N. A. Rahim, H. W. Ping, and M. N. Uddin, "Design Optimization and Analysis of AFPM Synchronous Machine Incorporating Power Density, Thermal Analysis, and Back-EMF THD," Progress In Electromagnetics Research, vol. 136, pp. 327-367, 2013.

[24] Y. C. Chong, E. J. E. Subiabre, M. A. Mueller, J. Chick, D. A. Staton, and A. S. McDonald, "The Ventilation Effect on Stator Convective Heat Transfer of an Axial-Flux Permanent-Magnet Machine," IEEE Transactions on Industrial Electronics, vol. 61, no. 8, pp. 4392-4403, August 2014.

[25] D. A. Howey, A. S. Holmes, and K. R. Pullen, "Measurement and CFD Prediction of Heat Transfer in Air-Cooled Disc-Type Electrical Machines," IEEE Transactions on Industry Applications, vol. 47, no. 4, pp. 1716-1723, July 2011.

[26] J. F. Gieras, R. J. Wang, and M. J. Kamper, Axial Flux Permanent Magnet Brushless Machines, 2nd ed. USA: Springer, 2008 .

Copyright $(\mathrm{C}$ by the authors. Licensee TAETI, Taiwan. This article is an open access article distributed under the terms and conditions of the Creative Commons Attribution (CC BY-NC) license (https://creativecommons.org/licenses/by-nc/4.0/). 